\title{
Shared genetic factors in migraine and depression
}

\author{
Evidence from a genetic isolate
}

A.H. Stam, MD

B. de Vries, MSc

A.C.J.W. Janssens, $\mathrm{PhD}$

K.R.J. Vanmolkot, $\mathrm{PhD}$

Y.S. Aulchenko, $\mathrm{PhD}$

P. Henneman, MSc

B.A. Oostra, PhD

R.R. Frants, PhD

A.M.J.M. van den

Maagdenberg, $\mathrm{PhD}$

M.D. Ferrari, MD, PhD

C.M. van Duijn, $\mathrm{PhD}$

G.M. Terwindt, MD,

$\mathrm{PhD}$

Address correspondence and reprint requests to Dr. Gisela M. Terwindt, Department of Neurology, Leiden University Medical Centre, Albinusdreef 2, PO Box 9600, 2300 RC Leiden, the Netherlands G.M.Terwindt@lumc.nl

Editorial, page 278

\section{ABSTRACT}

Objective: To investigate the co-occurrence of migraine and depression and assess whether shared genetic factors may underlie both diseases.

Methods: Subjects were 2,652 participants of the Erasmus Rucphen Family genetic isolate study. Migraine was diagnosed using a validated 3-stage screening method that included a telephone interview. Symptoms of depression were assessed using the Center for Epidemiologic Studies Depression scale and the depression subscale of the Hospital Anxiety and Depression Scale (HADS-D). The contribution of shared genetic factors in migraine and depression was investigated by comparing heritability estimates for migraine with and without adjustment for symptoms of depression, and by comparing the heritability scores of depression between migraineurs and controls.

Results: We identified 360 migraine cases: 209 had migraine without aura (MO) and 151 had migraine with aura (MA). Odds ratios for depression in patients with migraine were 1.29 (95\% confidence interval [Cl] 0.98-1.70) for $\mathrm{MO}$ and 1.70 (95\% Cl 1.28-2.24) for MA. Heritability estimates were significant for all migraine (0.56), $\mathrm{MO}(0.77)$, and $\mathrm{MA}(0.96)$, and decreased after adjustment for symptoms of depression or use of antidepressant medication, in particular for MA. Comparison of the heritability scores for depression between patients with migraine and controls showed a genetic correlation between HADS-D score and MA.

Conclusions: There is a bidirectional association between depression and migraine, in particular migraine with aura, which can be explained, at least partly, by shared genetic factors. Neurology ${ }^{\circledR} 2010 ; 74: 288-294$

\section{GLOSSARY}

CES-D = Center for Epidemiologic Studies Depression Scale; $\mathbf{C I}=$ confidence interval; ERF = Erasmus Rucphen Family; HADS-D = Hospital Anxiety and Depression Scale; IHS = International Headache Society; MA = migraine with aura; $\mathbf{M O}=$ migraine without aura; $\mathbf{O R}=$ odds ratio.

Migraine is a highly prevalent brain disorder that is characterized by recurrent headache attacks associated with nausea, vomiting, photophobia, and phonophobia (migraine without aura; $\mathrm{MO}$ ). In one third of migraineurs, attacks are preceded by transient focal neurologic symptoms (migraine with aura; MA). ${ }^{1}$ Migraineurs often have symptoms of depression. Population-based odds ratios (OR) range from 2.0 to 5.8 , with strongest associations for MA. ${ }^{2-13}$ The quality of life of migraineurs with depression is decreased. ${ }^{10,14}$ Comorbid depression is a risk factor for chronification of migraine ${ }^{15-17}$ and development of medication overuse headache. ${ }^{18}$ Patients with depression also have an increased risk for migraine, with risk estimates of 2.8-3.4 for migraine, 2.2 for $\mathrm{MO}$, and 4.0 for MA. ${ }^{2-4}$ This bidirectional relationship suggests that migraine and depression may share common etiologic factors. 
Twin- and family-based studies have shown that both migraine and depression have a strong genetic basis. ${ }^{19-24}$ Heritability estimates range from $0.33-0.53$ for migraine $33 \%-53 \%$ of the trait is explained by additive genetic effects) to $0.61-0.77$ for $\mathrm{MO}$ and 0.65-0.79 for MA. ${ }^{19-23}$ For depression, heritability estimates range from $0.17-0.78 .{ }^{24} \mathrm{Re}$ cent evidence suggests that both diseases share molecular pathways controlling serotonergic and glutaminergic neurotransmitter systems. ${ }^{25,26}$ Shared genetic factors may therefore underlie the bidirectional comorbidity of migraine and depression.

Here we determined the co-occurrence of migraine and depression in a Dutch genetic isolate, investigated to what extent genetic factors are involved in migraine, and investigated whether shared genetic factors may underlie the comorbidity of both disorders.

METHODS Study population. Subjects were participants of the Erasmus Rucphen Family (ERF) study, a family-based study in a genetically isolated community in the southwest of the Netherlands. ${ }^{27}$ In brief, the ERF study population includes 3,465 individuals ascertained based on genealogic background (not selected on phenotypes of interest), and are living descendants of 22 couples with at least 6 children baptized in the community church between 1850 and 1900. Founding couples are related through previous generations as demonstrated by extensive genealogic information from detailed church and municipal records. Hence, study participants were all members of a large extended pedigree. All individuals 18 years and older were invited to participate. Spouses were invited only for family members who had children 18 years and older.

Standard protocol approvals, registrations, and patients. The study was approved by the Medical Ethical Committee of the Erasmus MC Rotterdam and all participants gave written informed consent.

Clinical evaluation. Extensive clinical information from ERF participants $(n=3,465)$ was available. ${ }^{27}$ For this study, relevant clinical data were obtained by questionnaires (symptoms of headache and depression and education level) or from a visit to the research center (use of antidepressant medication).

Migraine. Migraineurs were identified between 2005 and 2007 using a 3-stage screening procedure assessing lifetime occurrence of migraine, which was previously validated in a populationbased study ${ }^{28}$ and which was based on the Classification Criteria of the International Headache Society (IHS). ${ }^{1}$ In brief, in the first stage, participants were asked to fill in 5 screening questions on headache and aura symptoms. Then, screen-positives received a detailed questionnaire on headache and aura symptoms. Finally, screen-positives were interviewed by telephone for further clarification of their answers. Subjects who were screen-positive but did not return the extensive headache questionnaire and subjects who could not be screened because they did not fill in or incompletely filled in the screening questions were directly con- tacted by telephone. Telephone interviews were performed by the principal study physician (A.H.S.), who is experienced in diagnosing migraine patients, and by well-trained medical students supervised by A.H.S. A final diagnosis was only made after the telephone interview and in consultation with a neurologist specialized in headache (G.M.T.).

Depressive symptoms. Symptoms of depression were assessed using the Center for Epidemiologic Studies Depression Scale (CES-D $)^{29}$ and the depression subscale of the Hospital Anxiety and Depression Scale (HADS-D).$^{30}$ Both scales are validated, reliable self-report measures of symptoms of depression. $^{31,32}$ The CES-D consists of 20 items with total scores ranging from 0 to 60 and the HADS-D of 7 items with scores ranging from 0 to 21 . Higher scores indicate more symptoms of depression. Depression scores were analyzed both as continuous variables and as dichotomous variables. For the dichotomous variable, depression was defined as a CES-D score $\geq 16^{31}$ and HADS-D score $\geq 8^{32}$ or the use of antidepressant medication. CES-D scores were missing for 327 and HADS-D for 104 subjects. Missing CES-D and HADS-D data were imputed based on data of the other depression scale, age, sex, and use of antidepressants using SPSS version 12.0, iterative expectationmaximization method. Depression scores were not imputed when both scores were missing.

Control group. The control group consisted of subjects who 1) did not report severe headache (pain severity score $\leq 4$ on a scale from 0 to 10 ); 2) did not report visual aura symptoms; 3 ) had never been diagnosed with migraine by a physician; and 4) never used specific antimigraine medication. Thus, subjects with mild headache in combination with visual aura are excluded from the control group. To exclude false negative control cases, we used an even more conservative definition for nonmigraineurs than in our previous population-based study. ${ }^{28}$

Statistical methods. For statistical analysis we used data from 3 migraine groups (i.e., all migraine cases, $\mathrm{MO}$, and MA) and the control group. General characteristics of the subjects of each group were compared using $\chi^{2}$ statistics for dichotomous variables and a Student $t$ test for continuous variables.

All analyses were corrected for inbreeding. The coefficient of inbreeding per individual was calculated based on available genealogic information using PEDIG software. ${ }^{33}$ This coefficient reflects the probability that 2 alleles at a given locus in an individual are identical by descent.

To examine the co-occurrence of migraine and depression, we calculated the OR for the risk of depression for each migraine group using multivariate mixed model regression analyses corrected for inbreeding, age, sex, and education and taking into account pedigree relations between individuals (polygenic liability threshold model). ${ }^{34}$

Mean CES-D and HADS-D scores were calculated for each group. $p$ Values comparing migraine subgroups vs controls were corrected for age, sex, level of education, use of antidepressants, inbreeding, and pedigree relations by multivariate linear mixed model regression, considering each migraine group as an independent variable and HADS-D or CES-D data as the continuous dependent variable. Because both CES-D data and HADS-D data deviated from the normal distribution, data were transformed by taking the natural logarithm (CES-D) and by taking the square root (HADS-D).

We calculated the heritability estimates $\left(b^{2}\right)$ as the ratio of the variance of a trait that is explained by additive polygenic effects to total phenotypic variance of the trait. The polygenic 


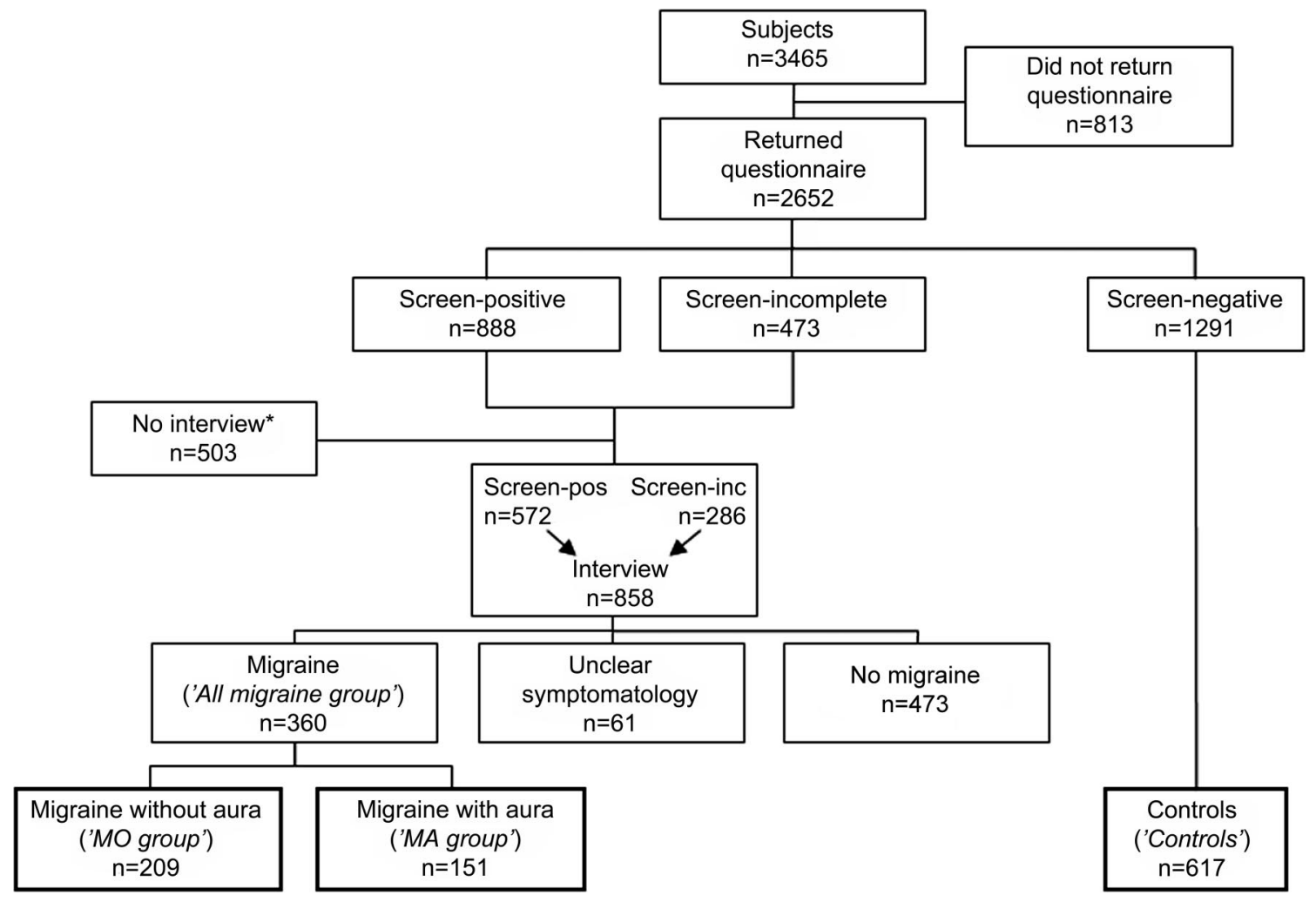

*Subjects were deceased or declined participation $(n=122)$, had an incorrect $(n=140)$ or unavailable $(n=10)$ telephone number, gave no permission for a telephone interview $(n=130)$, were not answering the telephone (at least 3 attempts were made) $(n=72)$, or indicated on the telephone that they did not want to cooperate with the interview $(n=29)$.

model was applied that assumes an infinite number of genetic factors with a small additive effect contributing to the trait variance. Analyses were adjusted for age, sex, and inbreeding. As sibship effects, a combination of effects induced by sharing early childhood environment and dominant genetic variation, did not significantly influence heritability estimation, this covariate was excluded from the analyses.

Involvement of shared genetic factors underlying migraine and depression was assessed by 2 complementary methods. First, heritability estimates for migraine were computed with and without depressive symptoms as covariate (i.e., HADS-D, CES-D scores and use of antidepressants included) (see also reference ${ }^{27}$ ). A difference between adjusted and unadjusted heritability estimates represents that part of the genetic component in migraine that is shared with depression.

Second, we compared the heritability of depression in patients with migraine to that in controls (i.e., assess whether a diagnosis of migraine influences the heritability of depression) by performing a bivariate polygenic analysis with both HADS-D and CES-D scores (i.e., depression score + migraine status, covariates: age, sex, inbreeding, use of antidepressant medication) (see for examples other references ${ }^{35,36}$ except here, we compared migraine vs no migraine status, instead of gender, to estimate the genetic correlation). The significance of a genetic correlation was determined using a likelihood ratio test.

Descriptive analyses were performed using SPSS version 12.0 for windows (SPSS, Chicago, IL). SOLAR 2.1.2 software package (Southwest Foundation for Biomedical Research, San Antonio, TX) was used for calculation of heritability estimates, regression analyses, and genetic correlations.
RESULTS Recruitment of patients with migraine. In total, 3,465 subjects participated in the ERF study. The figure shows the ascertainment flow chart of migraine cases. The questionnaire with headache screening questions was returned by 2,652 (76.5\%) subjects. Of these, 888 were screen-positive and 1,291 screen-negative; 473 subjects had not or only incompletely filled out the screening questionnaire. Of the screenpositives, $572(64.4 \%)$ subjects could be interviewed, of which 305 proved to have migraine. Of the group that was incompletely screened, 286 (60.5\%) subjects were interviewed, of which 55 proved to have migraine. A diagnosis could not be made in 503 subjects because of a variety of mainly logistic reasons. Of the 2,149 ERF participants who were interviewed $(\mathrm{n}=858)$ or who were screen-negative $(\mathrm{n}=1,291), 360$ (16.8\%) had migraine. Of these, 209 (58\%) had MO and $151(42 \%)$ had MA. A total of 617 subjects gave negative answers to all screening questions, thereby fulfilling the criteria for the control group.

Descriptive data. The demographic characteristics of all groups are shown in table 1 . No significant differences were found for the mean age. In line with the higher prevalence of migraine in women, more 


\begin{tabular}{|c|c|c|c|c|}
\hline \multicolumn{5}{|c|}{ Distribution of sex, age, and education in the various groups } \\
\hline Characteristics & $\begin{array}{l}\text { All migraine } \\
(n=360)\end{array}$ & $\begin{array}{l}\text { MO } \\
(n=209)\end{array}$ & $\begin{array}{l}\text { MA } \\
(n=151)\end{array}$ & $\begin{array}{l}\text { Controls } \\
(n=617)\end{array}$ \\
\hline Women, \% & $75^{\mathrm{a}}$ & $77^{\mathrm{a}}$ & $73^{a}$ & 47 \\
\hline Mean age, $y$ (SD) & $46.2(12.3)$ & $47.0(12.7)$ & $45.1(11.6)$ & 47.8 (15.3) \\
\hline \multicolumn{5}{|l|}{ Education, $\%^{c}$} \\
\hline Higher & $5^{b}$ & 5 & $4^{b}$ & 9 \\
\hline Medium & 64 & 63 & 66 & 65 \\
\hline Lower & 31 & 32 & 30 & 26 \\
\hline
\end{tabular}

Abbreviations: $\mathrm{MA}=$ migraine with aura; $\mathrm{MO}=$ migraine without aura. ${ }^{a} p<0.001$ compared to controls; ${ }^{b} p<0.05$ compared to controls.

${ }^{c}$ Education: higher $=$ college or university, medium $=$ secondary school or vocational technical training, lower = primary or elementary school or unfinished secondary school.

women were present in the migraine groups. Overall, patients with migraine, in particular those with MA, had lower education than controls.

Depression in migraineurs. Of the 2,652 participants who completed the questionnaire, HADS-D scores were obtained for 2,548 subjects and CES-D scores for 2,325. After imputation, HADS-D and CES-D scores were available for 2,584 subjects. Of these, 583 met our definition for depression. Of these, 91 were included in the migraine group and 82 in the nonmigraine control group. The remaining subjects were not included in the current assessment because they did not meet the inclusion criteria for either the all migraine or control group.

Twenty-five percent (91/360) of migraineurs and $13 \%(82 / 617)$ of controls were found to be depressed (table 2). Depression was more often seen in MA (32\%; 48/151) than in MO (21\%; 43/209). The ORs were 1.43 (95\% confidence interval [CI] $1.15-1.78)$ for all migraine, 1.29 (95\% CI $0.98-$ 1.70) for $\mathrm{MO}$, and 1.70 (95\% CI $1.28-2.24)$ for MA. Mean CES-D scores were higher $(p<0.001)$ in migraine patients compared to controls (all migraine: $12.0 \pm 10.2$, MO: $11.1 \pm 9.7$, MA: $13.2 \pm 10.7$, and controls: $7.5 \pm 7.4)$. The mean HADS-D score was higher $(p<0.01)$ in the all migraine and MA groups compared to controls (all migraine: $6.1 \pm 4.4$ and MA: $6.7 \pm 4.6$ ), but was not significantly differ-

\begin{tabular}{|llll|}
\hline Table 2 & \multicolumn{3}{l}{ Depression in migraine patients and controls } \\
Group & Depression, $n(\%)$ & OR $(95 \%$ Cl) & $p$ Value \\
Controls $(n=617)$ & $82(13)$ & 1.00 & - \\
MO $(n=209)$ & $43(21)$ & $1.29(0.98-1.70)$ & 0.07 \\
MA $(n=151)$ & $48(32)$ & $1.70(1.28-2.24)$ & $<0.001$ \\
\hline
\end{tabular}

Abbreviations: $\mathrm{Cl}=$ confidence interval; $\mathrm{MA}=$ migraine with aura; $\mathrm{MO}=$ migraine without aura; $\mathrm{OR}=$ odds ratio.

aPrevalence of depression per group is given. Depression was defined as scoring both Center for Epidemiologic Studies Depression Scale $\geq 16$ and Hospital Anxiety and Depression Scale $\geq 8$ or use of antidepressants. Odds ratios for the risk of depression in migraine patients are adjusted for sex, age, education, and inbreeding. ent between the $\mathrm{MO}$ and control group (MO: $5.7 \pm$ 4.2 and controls: $4.9 \pm 3.7$ ).

Heritability estimates of migraine. In total, $325 \mathrm{mi}-$ graine patients (188 MO and $137 \mathrm{MA}$ ) and 562 control subjects were present in the ERF extended pedigree. ${ }^{27}$ Heritability estimates were $0.56(95 \% \mathrm{CI}$ $0.26-0.86)$ for all migraine, 0.77 (95\% CI $0.38-$ $1.00)$ for $\mathrm{MO}$, and 0.96 (95\% CI $0.51-1.00)$ for MA (table 3). These estimates decreased after adjustment for depressive symptoms to 0.51 (95\% CI $0.19-0.83)$ for all migraine, 0.75 (95\% CI 0.32 1.00) for $\mathrm{MO}$, and 0.81 (95\% CI $0.31-1.00)$ for MA. The decrease was highest in patients with MA (15\%), compared to a decrease of $4 \%$ in the all migraine group and $2 \%$ in MO patients. This would suggest that shared genetic factors particularly underlie the comorbidity of depression and MA.

Next we compared the heritability for depression in migraineurs and controls. A correlation of 1 indicates that there is no difference in the genetics of depression between patients and controls, while a deviation from 1 suggests shared genetic factors for depression and migraine. Only for HADS-D and MA a reduced genetic correlation was found (correlation of 0.36) indicating that genetic factors causing symptoms of depression in MA patients differ from those involved in depression in controls. In MO patients, the correlation coefficient was 1 , indicating no difference in genetic factors for depression in MO and controls. For CES-D, there was no difference seen between migraine patients and controls.

DISCUSSION We investigated the co-occurrence of migraine and depression in a large Dutch genetic isolate and to what extent shared genetic factors are involved. Our study is particularly suited to address these questions because of the following reasons: 1 ) our 3-step diagnostic procedure, including a telephone interview for clarification and confirmation of the clinical symptoms and a final diagnosis according to the Classification Criteria of the IHS, ${ }^{1}$ guaranteed a highly reliable diagnosis of migraine; 2) the presence of depression was assessed using 2 different depression scales; 3) our study population includes a very large number of well-characterized subjects covering a broad age range from 18 to 91 years.

We found an increased risk of depression and depressive symptoms in migraine patients, in particular in those with aura. These findings are in agreement with previous studies from outbred populations. ${ }^{2-13}$ ORs for depression in migraine patients in those studies were larger (2.0-5.8 for MA) than ORs observed here (1.7 for MA), which may be due to differences in the diagnostic methods and definitions. This seems particularly true for depression. While 29 
Table $3 \quad$ Heritability estimates of migraine with and without adjustment for depression

$\begin{array}{llll} & h^{2}(95 \% \mathrm{Cl})^{\mathrm{a}} & \begin{array}{l}h^{2}(95 \% \mathrm{Cl})(\text { with } \\ \text { adjustment for } \\ \text { depression })^{\mathrm{b}}\end{array} & p \text { Value } \\ \text { All migraine }(\mathrm{n}=325) & 0.56(0.26-0.86)^{\mathrm{d}} & 0.51(0.19-0.83)^{\mathrm{d}} & 0.81 \\ \text { MO }(\mathrm{n}=188) & 0.77(0.38-1.00)^{\mathrm{d}} & 0.75(0.32-1.00)^{\mathrm{d}} & 0.95 \\ \text { MA }(\mathrm{n}=137) & 0.96(0.51-1.00)^{\mathrm{d}} & 0.81(0.31-1.00)^{\mathrm{e}} & 0.65\end{array}$

Abbreviations: $\mathrm{Cl}=$ confidence interval; $\mathrm{MA}=$ migraine with aura; $\mathrm{MO}=$ migraine without aura.

aHeritability estimates are based on a polygenic model, covariates: age, sex, and inbreeding coefficient.

bHeritability estimates are based on a polygenic model, covariates: age, sex, and inbreeding coefficient, Hospital Anxiety and Depression Scale, Center for Epidemiologic Studies Depression Scale, use of antidepressant medication.

${ }^{c} p$ Value for the difference in heritability estimates before and after adjustment for depression. $p$ Values for heritability estimates: ${ }^{d} p<0.001$ and ${ }^{e} p<0.01$

several studies addressed lifetime prevalence of depression, ${ }^{2,4,5,9}$ which is notoriously difficult to assess reliably, we studied current depression, which is reliable to diagnose and will result in lower prevalence data. One might thus argue that presence of lifetime depression was underestimated in our study.

Some $23.5 \%$ of the 3,465 subjects were nonresponders, which may have introduced a selection bias leading to an overrepresentation of very depressed patients or severe migraine patients in the nonresponder population. However, such a bias in this particular study is less likely, as we used a general questionnaire designed to collect information on a large number of traits, not only related to headache and depression.

Heritability estimates in our study were significant for all 3 migraine groups and were substantially higher for MA than for MO. This is in accordance with studies in outbred populations, ${ }^{19-23}$ confirming the hypothesis that the genetic contribution in MA is stronger than in MO. Also in line with these studies, a lower heritability estimate was observed for all migraine than for MO or MA, which indicates that MA and $\mathrm{MO}$ share some, but not all, genetic factors. We observed a remarkably high heritability for MA of over $90 \%$ in ERF. This suggests that most of the variance in MA in ERF is explained by genetic factors. This finding may be particularly relevant for gene discovery and suggests that MA is the most promising migraine subtype to search for migraine genes. Moreover, our observation that heritability estimates for MA decreased when adjusting for depression indicates that shared genetic factors may underlie depression and MA. For $\mathrm{MO}$, a smaller decrease in heritability estimates was observed, suggesting that a small fraction of the comorbidity with depression is explained by shared genetic factors for this migraine subtype.

In our bivariate analysis, we found remarkably different results when using the 2 depression scales.
When depression was determined by using the HADS-D scale, we found evidence for shared genetic factors for migraine with aura and depression. However, no such association was observed when depression was determined using the CES-D scale. This discrepancy may seem remarkable as both scales have been validated for the assessment of symptoms of depression $^{31,32}$ and there is a high correlation for both depression scales in our study (Pearson correlation coefficient rho $=0.75 ; p<0.001)$. However, both scales assess slightly different symptoms, which may well explain our findings. As the HADS-D was specifically designed to prevent noise signal from somatic disorders, ${ }^{32}$ it excludes physical symptoms of depression, such as insomnia, fatigue, or loss of appetite. In the CES-D scale, however, these symptoms are included. Although difficult to prove, we suggest that these physical symptoms are more likely a consequence of the migraine attacks than due to depression. Consequently, we propose that the CES-D score, in a way, is confounded by migraine-related symptoms and therefore less suited to assess shared genetic factors in migraine and depression.

Previous studies found a bidirectional association between migraine, in particular those with aura, and depression..$^{2-4}$ This suggests that common pathogenetic pathways, at least partly, underlie both disorders, rather than that one is the consequence of the other. Thus, migraine patients may develop depression as a result of the demoralizing experience of recurrent and disabling headaches. However, then a correlation with disease disability would be expected, which was not found in previous studies. ${ }^{2}$ Also, an even stronger association with MO should be seen, which in general is the more disabling form of migraine $^{1}$ and usually associated with higher attack frequencies. Our study provides evidence that this bidirectional relationship can be explained, at least partly, by shared underlying genetically determined disease mechanisms. Support for our findings comes from a recent twin study that also suggested that shared genetic risk factors may underlie migraine and depression. ${ }^{37}$ Unfortunately that study has important limitations. First of all, it is unclear whether a correct migraine diagnosis was made as a diagnosis was based on "self-reported physician's diagnosis" and not on well-defined IHS criteria. A similar criticism can be made with respect to the diagnosis of depression. This may well have led to misclassification and underreporting of migraine and depression. Second, unlike in our study, the sample consisted of female subjects only, and results therefore cannot be extrapolated to male patients. Third, no distinction was made between MA and MO. This last limitation is 
very relevant in light of our observation that shared genetic effects between migraine and depression are particularly evident for migraine with aura.

Identification of common genetic factors may significantly improve insight into the molecular basis of these common and highly disabling episodic brain disorders.

\section{AUTHOR CONTRIBUTIONS}

Statistical analysis was conducted by Dr. Yurii S. Aulchenko.

\section{DISCLOSURE}

Dr. Stam has received independent research support from NWO. B. de Vries reports no disclosures. Dr. Janssens serves on the editorial boards of Plos ONE, Medical Decision Making, and Public Health Genomics. Dr. Vanmolkot, Dr. Aulchenko, P. Henneman, Dr. Oostra, Dr. Frants, and Dr. van den Maagdenberg report no disclosures. Dr. Ferrari has, in the past 3 years, received grants and consultancy or industry support from Almirall, Coherex, Colucid, Eisai, GlaxoSmithKline, Linde, MAP, Medtronic, Menarini, Merck Sharp \& Dohme, Minster, Pfizer, and St Jude and independent support from NWO, the NIH (R01 NS61382-01 (PI), European Community FP6, Biomed EC, and the Dutch Heart Foundation. Dr. van Duijn reports no disclosures. Dr. Terwindt received consultancy or industry support from Merck Sharp \& Dohme and Janssen-Cilag and independent support from NWO.

Received April 2, 2009. Accepted in final form August 24, 2009.

\section{REFERENCES}

1. Headache Classification Subcommittee of the International Headache Society. The International Classification of Headache Disorders, 2nd ed. Cephalalgia 2004;24:1-160.

2. Breslau N, Lipton RB, Stewart WF, Schultz LR, Welch KM. Comorbidity of migraine and depression: investigating potential etiology and prognosis. Neurology 2003;60:1308-1312.

3. Breslau N, Davis GC, Schultz LR, Peterson EL. Joint 1994 Wolff Award Presentation: Migraine and major depression: a longitudinal study. Headache 1994;34:387-393.

4. Breslau N, Schultz LR, Stewart WF, et al. Headache and major depression: is the association specific to migraine? Neurology 2000;54:308-313.

5. Kecceci H, Dener S, Analan E. Co-morbidity of migraine and major depression in the Turkish population. Cephalalgia 2003;23:271-275.

6. McWilliams LA, Goodwin RD, Cox BJ. Depression and anxiety associated with three pain conditions: results from a nationally representative sample. Pain 2004;111:77-83.

7. Merikangas KR, Angst J, Isler H. Migraine and psychopathology: results of the Zurich cohort study of young adults. Arch Gen Psychiatry 1990;47:849-853.

8. Patel NV, Bigal ME, Kolodner KB, et al. Prevalence and impact of migraine and probable migraine in a health plan. Neurology 2004;63:1432-1438.

9. Swartz KL, Pratt LA, Armenian HK, Lee LC, Eaton WW Mental disorders and the incidence of migraine headaches in a community sample: results from the Baltimore Epidemiologic Catchment area follow-up study. Arch Gen Psychiatry 2000;57:945-950.

10. Lipton RB, Hamelsky SW, Kolodner KB, Steiner TJ, Stewart WF. Migraine, quality of life, and depression: a population-based case-control study. Neurology 2000;55: $629-635$.
11. Oedegaard KJ, Neckelmann D, Mykletun A, et al. Migraine with and without aura: association with depression and anxiety disorder in a population-based study: The HUNT Study. Cephalalgia 2006;26:1-6.

12. Zwart JA, Dyb G, Hagen K, et al. Depression and anxiety disorders associated with headache frequency: The NordTrondelag Health Study. Eur J Neurol 2003;10:147-152.

13. Camarda C, Pipia C, Taglialavori A, et al. Comorbidity between depressive symptoms and migraine: preliminary data from the Zabut Aging Project. Neurol Sci 2008;suppl 1:S149-S151.

14. Lantéri-Minet M, Radat F, Chautard MH, Lucas C. Anxiety and depression associated with migraine: influence on migraine subjects' disability and quality of life, and acute migraine management. Pain 2005;118:319-326.

15. Bigal ME, Lipton RB. Modifiable risk factors for migraine progression. Headache 2006;46:1334-1343.

16. Wang SJ, Juang KD, Fuh JL, Lu SR. Psychiatric comorbidity and suicide risk in adolescents with chronic daily headache. Neurology 2007;68:1468-1473.

17. Mercante JP, Peres MF, Guendler V, Zukerman E, Bernik MA. Depression in chronic migraine: severity and clinical features. Arq Neuropsiquiatr 2005;63:217-220.

18. Radat F, Créac'h C, Swendsen JD, et al. Psychiatric comorbidity in the evolution from migraine to medication overuse headache. Cephalalgia 2005;25:519-522.

19. Gervil M, Ulrich V, Kaprio J, Olesen J, Russell MB. The relative role of genetic and environmental factors in migraine without aura. Neurology 1999;53:995-999.

20. Russell MB, Iselius L, Olesen J. Inheritance of migraine investigated by complex segregation analysis. Hum Genet 1995;96:726-730.

21. Ulrich V, Gervil M, Kyvik KO, Olesen J, Russell MB. The inheritance of migraine with aura estimated by means of structural equation modelling. J Med Genet 1999;36:225227.

22. Mulder EJ, Van Baal C, Gaist D, et al. Genetic and environmental influences on migraine: a twin study across six countries. Twin Res 2003;6:422-431.

23. Ziegler DK, Hur YM, Bouchard TJ, Jr., Hassanein RS, Barter R. Migraine in twins raised together and apart. Headache 1998;38:417-422.

24. Sullivan PF, Neale MC, Kendler KS. Genetic epidemiology of major depression: review and meta-analysis. Am J Psychiatry 2000;157:1552-1562.

25. Pietrobon D. Migraine: new molecular mechanisms. Neuroscientist 2005;11:373-386.

26. Muller N, Schwarz MJ. The immune-mediated alteration of serotonin and glutamate: towards an integrated view of depression. Mol Psychiatry 2007;12:988-1000.

27. Sleegers K, de Koning I, Aulchenko YS, et al. Cerebrovascular risk factors do not contribute to genetic variance of cognitive function: the ERF study. Neurobiol Aging 2007; 28:735-741.

28. Launer LJ, Terwindt GM, Ferrari MD. The prevalence and characteristics of migraine in a population-based cohort: the GEM study. Neurology 1999;53:537-542.

29. Radloff LS. The CES-D Scale: a self-report depression scale for research in the general population. Applied Psychological Measurement 1977;1:385-401.

30. Zigmond AS, Snaith RP. The hospital anxiety and depression scale. Acta Psychiatr Scan 1983;67:361-370.

31. Weissman MM, Sholomskas D, Pottenger M, Prusoff BA, Locke BZ. Assessing depressive symptoms in five psychiat- 
ric populations: a validation study. Am J Epidemiol 1977; 106:203-214.

32. Bjelland I, Dahl AA, Haug TT, Neckelmann D. The validity of the Hospital Anxiety and Depression Scale: an updated literature review. J Psychosom Res 2002;52:69-77.

33. Boichard D. PEDIG, a FORTRAN package for pedigree analysis studied for large populations. Paper presented at Proceeding of the 7 th World Congress, Montpelier, France, August 19-23, 2002.

34. Almasy L, Blangero J. Multipoint quantitative-trait linkage analysis in general pedigrees. Am J Hum Genet 1998;62: $1198-1211$.
35. Henneman P, Aulchenko YS, Frants RR, van Dijk KW, Oostra BA, van Duijn CM. Prevalence and heritability of the metabolic syndrome and its individual components in a Dutch isolate: the Erasmus Rucphen Family study. J Med Genet 2008;45:572-577.

36. Pan L, Ober C, Abney M. Heritability estimation of sexspecific effects on human quantitative traits. Genetic Epidemiol 2007;31:338-347.

37. Schur EA, Noonan C, Buchwald D, Goldberg J, Afari N. A twin study of depression and migraine: evidence for shared genetic vulnerability. Headache 2009;49:14931502.

\section{AAN Membership is Your Ticket to Annual Meeting Savings}

If you're planning on attending the $62^{\text {nd }}$ American Academy of Neurology Annual Meeting in Toronto, April 10 through 17, 2010, you should know that an AAN membership can save you nearly $\$ 400$ on Annual Meeting registration, program fees, and education products-that's just about the cost of membership dues alone!

On top of that, AAN membership provides additional benefits and savings you can enjoy throughout the year, such as deep discounts on education programs and products, tools for your practice, free advocacy training opportunities, free subscriptions to Neurology ${ }^{\circledR}$ and Neurology Today ${ }^{\circledR}$, and much more.

Renew your 2010 membership today at www.an.com/dues or call (800) 879-1960 or (651) 6951940 (international)! Early registration deadline ends March 10, 2010.

*Ifyou are not an AAN member, join today! Visit www.aan.com/membership or call (800) 879-1960 or (651) 695-1940 (international) to become a member and take advantage of the member-only discounts on Annual Meeting registration and course fees. 